\title{
Scientific and Practical Conference "Modern Biological Methods for Reproductive Medicine" (February 25, 2009, Moscow, Russia)
}

DOI: $10.1134 / \mathrm{S} 1062360409060101$

The scientific and practical conference on the modern biological methods for reproductive medicine was held in the Kol'tsov Institute of Developmental Biology, Russian Academy of Sciences, on February 25th, 2009.

The goals of this conference were to expand the modern concepts of the pathogenesis of some diseases affecting the reproductive system, assist in introduction of the state-ofthe-art results of molecular biological and cellular studies to the diagnostics of fertility abnormalities, and increase the efficiency of infertility therapy and reproductive technologies.

The presentation by L.F. Kurilo (Medical Genetic Research Center of the Russian Academy of Medical Sciences) considered the genetic mechanisms underlying morphogenetic and functional pathologies of the reproductive system and the opportunities offered for diagnostics and prevention of various pathologies.

E.E. Bragina (Belozersky Institute of Physicochemical Biology, Moscow State University) presented the results of ultrastructural studies of human spermatozoids. Quantitative ultrastructural analysis of the chromatin in spermatozoids demonstrates that a $28 \%$ rate of spermatozoids with abnormal chromatin condensation is critical for the development of an embryo. Bragina also showed the data on the intragametic herpes infection of human spermatozoids and that an abnormality in the centriole structure could cause idiopathic impairment of embryo development.

The topic of presentation by A.A. Kushch (Ivanovsky Institute of Virology, Russian Academy of Medical Sciences) was the study of the rate of detection of the herpes simplex virus (HSV) in the sperm ejaculate of men with impaired fertility and the evidence for a negative HSV effect on sperm quality. HSV is significantly more frequently detected in both the whole ejaculate and the fraction of actively moving spermatozoids of the man cohort with primary infertility as compared with practically healthy individuals. Infective HSV can be released in the case of an asymptomatic herpes virus infection.

The lecture of a group of authors (A.Yu. Kulibin, T.L. Marshak, and S.T. Zakhidov; Kol'tsov Institute of Developmental Biology, Russian Academy of Sciences, and Moscow State University) dwelled on the modern concepts in the biology of spermatogonial stem cells
(SSC) and Sertoli cells (SSC niche) and illustrated successful development of the methods for SSC isolation, cultivation, and transplantation, offering great opportunities for practical medicine, construction of transgenic animals, preservation of rare and endangered species, and in vitro modeling of spermatogenesis.

S.A. Yakovenko (Physical Faculty, Moscow State University) presented a developed original microscopic device providing for resolving low-contrast vacuoles with a size of about $0.1 \mu \mathrm{m}$ in the spermatozoid head. Use of spermatozoids without vacuoles considerably increases the rate of successful pregnancies when using one of the reproductive technologies---intracytoplasmic injection of spermatozoids into the ovum.

After hearing and discussing the presentations, the conference participants developed recommendations underlining that

(1) It is necessary to develop the standards (protocols) for combined examination of the patients with reproductive pathologies using state-of-the-art genetic, cytological, ultrastructural, and molecular biological techniques;

(2) It is purposeful to use ultrastructural, virological, and cytogenetic analyses of male gametes in the case of miscarriages and unsuccessful application of reproductive technologies;

(3) It is purposeful to discuss the topical problems connected with the deficiency in medical geneticists and the staff of some other specialties required for complex diagnostic and research procedures (cytogenetics), because biologists cannot attend certification courses on a regular basis; and

(4) The scientific developments in the field of diagnostics, therapy, and prevention of abnormalities in the reproductive health under the national project of demographic development in Russia are a priority direction.

It was proposed to organize on a regular basis the conferences that would inform reproductologists, gynecologists, andrologists, and laboratory staff working in practical medicine about the relevant modern biological methods.

$$
\text { V.Yu. Polyakov }
$$

E-mail:vladimirpolyakov@mail.ru 